\title{
Retraction: Effects of synthetic magnetic flux in a parity-time-symmetric system of three coupled optical resonators [Phys. Rev. A 96, 043821 (2017)]
}

\author{
L. Jin and F. Xin \\ (Received 4 November 2017; published 16 November 2017)
}

DOI: 10.1103/PhysRevA.96.059905

The authors recently have found a flaw in the basic modeling of the system presented in this paper. The gain as a result of lasing is nonlinear and depends on the clockwise and counterclockwise mode intensities; the clockwise and counterclockwise modes experience opposite magnetic fluxes. Therefore, the linear equations (1)-(3) in the paper described only one type of mode and cannot characterize the real physics of the system. Since the equations are critical to this paper, we wish to retract this paper to avoid misleading the readership. We regret the error. 